\title{
TYPE 1 FUZZY FUNCTION APPROACH BASED ON RIDGE REGRESSION
}

\section{FOR FORECASTING}

Fuzzy function approach is a kind of fuzzy inference system that can produce successful results for analyzing of forecasting problems. In a fuzzy function approach, a fuzzy function corresponding to each fuzzy set is generated by using multiple regression analysis. The number of explanatory variables in multiple regression analysis is increased via the non-linear transformations of the membership functions to improve the prediction performance of the model. In a fuzzy function approach, it can be found a high correlation between the non-linear transformations of membership functions and therefore the multiple linear regression method used to define fuzzy functions, which has multicollinearity issues. In order to overcome this problem, a new fuzzy function approach using ridge regression instead of multiple linear regression in Type 1 fuzzy function approach is proposed in this paper. The new Type 1 approach is applied to various real world time series data and the results are compared to the ones obtained by other techniques. The results obtained with the new methodology show superior forecasts performance.

Keywords: Type 1 fuzzy function approach, ridge regression, fuzzy inference system, forecasting, fuzzy c-means.

\section{INTRODUCTION}

The future planning has become very important in the world we live in. It is vital to obtain accurate and reliable forecasts in making plans for the future. Fuzzy inference systems have been added in recent years to the methods used to obtain predictions. Fuzzy inference systems work based on fuzzy sets created for input and output data set on hand. In the literature, the fuzzy inference system proposed by Takagi and Sugeno (1985) and the adaptive network fuzzy inference system (ANFIS) proposed by Jang (1993) have been commonly used for forecasting problem. Both of these fuzzy inference systems work based on a rule base. The determination of these rules requires expert knowledge and this situation prevents the objective working of the inference system.

Therefore, fuzzy function approach was proposed by Turksen (2008). Fuzzy function approach does not need rule base as it uses fuzzy functions instead of rules. The different applications of fuzzy function approach were developed by Celikyilmaz and Turksen (2008a, b) and Turksen (2009). The detailed information about fuzzy functions can be found in Celikyilmaz and Turksen (2009). A hybrid fuzzy function approach was proposed by Zarandi et al. (2013). The fuzzy function approach for forecasting problem was reconsidered in the study of Beyhan and Alci (2010).

Fuzzy function can produce successful results for prediction problems. In a fuzzy function approach, while obtaining fuzzy functions, the input matrix is extended with the use of various nonlinear transformations of memberships. It should not be a significant linear relationship between the explanatory variables in multiple regression method used in the obtaining of fuzzy functions. Linear relationship between the explanatory variables in multiple regression leads to multicollinearity problem and this situation causes an increase in variance of estimators and to obtain inconsistent prediction results. It is clearly seen that the nonlinear functions of the memberships used to obtain fuzzy functions are related between each other.

As a result, Type 1 fuzzy function (T1FF) approach works based on regression models with multicollinearity problem. The presence of multicollinearity problem in T1FF method is a sign that the results obtained from T1FF method are not reliable. In order to obtain reliable results in T1FF method, 
multicollinearity problem has to be eliminated. For this purpose, ridge regression technique can be used instead of classic regression analysis to overcome multicollinearity problem.

Ridge regression was firstly proposed by Hoerl and Kennard (1970a) and it has been frequently used in the literature to overcome multicollinearity problem. There are many methods to determine multicollinearity problem. One of these methods is to determine shrinkage parameter in ridge regression. A simple formula to obtain shrinkage parameter in ridge regression was proposed by Hoerl et al. (1975).

In this paper, the multicollinearity problem in T1FF approach is solved by using ridge regression technique. The proposed new T1FF approach is called as "Type 1 fuzzy function approach based on ridge regression (T1FFRR)". In the proposed method, the shrinkage parameter of the ridge regression was obtained as in the study of Hoerl et al. (1975). The new T1FFRR approach is applied to many realworld time series data sets and the results are compared to the ones obtained from other techniques.

The rest part of the paper can be outlined as below: The second section of the paper is about T1FF approach. Ridge regression technique is briefly summarized in Section 3. In the fourth section of the paper, the proposed new T1FFRR approach is introduced with details. Section 5 presents the results from the application of the proposed method to real life data sets and finally Section 6 presents conclusions and discussions.

\section{TYPE 1 FUZZY FUNCTION APPROACH}

Fuzzy inference system proposed by Takagi and Sugeno (1985) and ANFIS are fuzzy inference systems that require the creation of a rule base. Fuzzy function approach proposed by Turksen (2008) is a fuzzy inference system working without a rule base. In a fuzzy function approach, a linear function is composed via linear regression method for each fuzzy set obtained from fuzzy c-means and the output of the system is obtained from the output of weighted fuzzy function predictions with memberships as weights. Four different fuzzy function approaches were given in Celikyilmaz and Turksen (2009). In a fuzzy function approach, fuzzy function is obtained as the number of clusters and requires fewer parameters than other fuzzy inference systems. The non-linear relationship between input and output can be modeled on the grounds that fuzzy function approach is an approach based on data. The most important issue in fuzzy function approach is that it has multicollinearity problem and also there is no method in the literature that provides a solution on this subject. In this study, Type 1 fuzzy function approach has been modified by solving multicollinearity problem.

\section{RIDGE REGRESSION}

In the regression analysis, the presence of full or high level relationships among the independent variables is explained by the concept of multicollinearity. The presence multicollinearity has significant effects on the estimates of least squares of the regression coefficients. The most important effect of multicollinearity problem is that the regression coefficients cause the smallest squares estimates to have large variance. That means if there is multicollinearity among the independent variables, it would be inappropriate to do solutions with ordinary least squares (OLS).

It is well known that the OLS estimates of regression coefficients $(\hat{\beta})$ are unbiased estimators. In the ridge regression method, different from classical regression analysis, the ridge estimates of regression coefficients are biased estimators. The ridge model estimates the coefficients in the regression analysis as biased to reduce the variances of the predictions.

Ridge regression was firstly proposed by Hoerl and Kennard (1970a). In the literature, several methods have been developed to determine whether there is multicollinearity or not. One of these methods is to 
examine correlation coefficients. If the correlation coefficients between the independent variables are close to 1 , this indicates the presence of multicollinearity problem. One of the other methods to determine the multicollinearity problem is variance inflation factors $(\boldsymbol{V I F})$. The diagonal elements of $\operatorname{Var}(\widehat{\beta})$ is called as $\boldsymbol{V I F}$. If $\boldsymbol{V I F}$ value is bigger than 10, this indicates the presence of multicollinearity problem.

The OLS estimates of regression coefficients and ridge estimates of regression coefficients are given in the Equations 1 and 2 respectively.

$$
\begin{aligned}
& \hat{\beta}=\left(X^{\prime} X\right)^{-1} X^{\prime} Y \\
& \hat{\beta}_{R}=\left(X^{\prime} X+k I\right)^{-1} X^{\prime} Y
\end{aligned}
$$

One of the main obstacles in using ridge regression is the choice of value of $\boldsymbol{k}$.This $\boldsymbol{k}$ value is also called as biased parameter or shrinkage parameter and it takes values between 0 and 1 . In the literature there are some methods to find this $\boldsymbol{k}$ value. One of these methods is ridge trace method proposed by Hoerl and Kennard (1970). In this method, Hoerl and Kennard (1970) suggested using a graphic to find this k value. Besides this method, in the advancing years, Hoerl et al. (1975) proposed an iterative method for selecting of the value of $\mathrm{k}$. This method is based on the Equation 3.

$$
k=\frac{p \widehat{\sigma}^{2}}{\widehat{\beta}, \widehat{\beta}}
$$

\section{THE PROPOSED METHOD}

In $\mathrm{T} 1 \mathrm{FF}$ approach, ridge regression technique can be used to eliminate the multicollinearity problem that arises from the relationship between non-linear transformations of the membership values. The forecasting performance will be increased in a fuzzy function approach which does not have the multicollinearity issue.

A new T1FFRR approach that uses ridge regression to define fuzzy functions is given as an algorithm below.

\section{Algorithm: The Proposed Method}

Step 1. The inputs of the system are lagged variables (number of $p$ ).

The model order $(p)$ is determined according to the structure of the time series. Time series is shown as column vectors in the form of $X_{t}=\left[x_{1}, x_{2}, \ldots, x_{n}\right]^{\prime}$. The matrix $\mathrm{Z}$ is composed of the input and outputs of the system and its dimension is $(n-p) \times(p+1)$.

$$
Z=\left[\begin{array}{lllll}
X_{t} & X_{t-1} & X_{t-2} & \ldots & X_{t-p}
\end{array}\right]
$$

The elements of the matrix are clustered using Fuzzy Clustering Method (FCM) technique proposed by Bezdek (1981). FCM clustering technique is applied iteratively as per Equations (7) and (8). In these Equations, $c, \quad v_{i} \quad(i=1,2, \ldots, c)$ and $\mu_{i k}(i=1,2, \ldots, c ; k=1,2, \ldots, n)$ represent the number of fuzzy sets, cluster centers and membership values, respectively.

$$
\begin{aligned}
& v_{i}=\frac{\sum_{k=1}^{n}\left(\mu_{i k}\right)^{f} z_{k}}{\sum_{k=1}^{n}\left(\mu_{i k}\right)^{f}}, i=1,2, \ldots, c \\
& \mu_{i k}=\left[\sum_{j=1}^{c}\left(\frac{d\left(z_{k}, v_{i}\right)}{d\left(z_{k}, v_{j}\right)}\right)^{\frac{2}{f-1}}\right]^{-1}, i=, 2, \ldots, c ; k=1,2, \ldots, n
\end{aligned}
$$


where $f$ is the degree of fuzziness, $z_{k}$ is a vector whose elements are the elements compose of $k^{\text {th }}$ row of $Z$. $\mu_{i k}$; is the degree of belongingness of $k^{\text {th }}$ observation to $i^{\text {th }}$ cluster and $d(z, v)$ : is Euclidian distance and is computed by using Equation 9.

(9)

$$
d\left(z_{k}, v_{i}\right)=\left\|z_{k}-v_{i}\right\|
$$

Step 2. The membership values are determined by using the Equation 8 for the input data set according to the center determined from FCM technique.

$$
\mu_{i k}=\left[\sum_{j=1}^{n}\left(\frac{d\left(x_{k}, v_{i}\right)}{d\left(x_{k}, v_{j}\right)}\right)^{\frac{2}{f i-1}}\right]^{-1}, i=1,2, \ldots, c ; k=1,2, \ldots, n
$$

where; $x_{k}$, is a row of the input matrix which is generated for lagged variables and it is a vector whose elements are the elements compose of $k^{\text {th }}$ row and this value is taken as zero when $\mu_{i k} \leq \alpha$. $f_{i}$ is fuzziness index.

$$
X=\left[\begin{array}{llll}
X_{t-1} & X_{t-2} & \ldots & X_{t-p}
\end{array}\right]
$$

Step 3. For each cluster $i$, the membership values of each input data sample $\left(\mu_{i k}\right)$, non-linear transformations of membership values and original inputs are used as explanatory variables and $i^{t h}$ fuzzy function is obtained from predicting of $\boldsymbol{Y}^{(i)}=\boldsymbol{X}^{(i)} \boldsymbol{\beta}^{(i)}+\boldsymbol{\varepsilon}^{(i)}$ multiple regression model. $X^{(i)}$ and $Y^{(i)}$ matrices are as follows when the number of lagged variables is $p$ and using transformations $\mu_{i 1}{ }^{2}, \exp \left(\mu_{i 1}\right)$ and $\ln \left(\left(1-\mu_{i 1}\right) / \mu_{i 1}\right)$ for non-linear transformation of the membership values.

$$
\begin{gathered}
\boldsymbol{X}^{(i)}=\left[\begin{array}{ccccccc}
\mu_{i 1} & \mu_{i 1}{ }^{2} & \exp \left(\mu_{i 1}\right) & \ln \left(\left(1-\mu_{i 1}\right) / \mu_{i 1}\right) & x_{1} & \cdots & x_{p} \\
\mu_{i 2} & \mu_{i 2}{ }^{2} & \exp \left(\mu_{i 2}\right) & \ln \left(\left(1-\mu_{i 2}\right) / \mu_{i 2}\right) & x_{2} & \cdots & x_{p+1} \\
\vdots & \vdots & \vdots & \vdots & \vdots & \cdots & \vdots \\
\mu_{i n} & \mu_{i n}{ }^{2} & \exp \left(\mu_{i n}\right) & \ln \left(\left(1-\mu_{i n}\right) / \mu_{i n}\right) & x_{n-p} & \cdots & x_{n-1}
\end{array}\right] \\
\boldsymbol{Y}^{(i)}=\left[\begin{array}{c}
x_{p+1} \\
x_{p+2} \\
\vdots \\
x_{n}
\end{array}\right]
\end{gathered}
$$

(13)

Maximum likelihood estimators of regression parameters are obtained as follows.

$$
{\widehat{\beta_{R}}}^{(i)}=\left(\boldsymbol{X}^{(i)^{\prime}} \boldsymbol{X}^{(i)}+k I\right)^{-1} \boldsymbol{X}^{(i)}{ }^{\prime} \boldsymbol{Y}^{(i)}
$$

$$
\widehat{\boldsymbol{Y}}^{(i)}=\boldsymbol{X}^{(i)}{\widehat{\beta_{R}}}^{(i)}
$$

Here shrinkage parameter $(\boldsymbol{k})$ is obtained by using Equation 4. 
Step 4. The results obtained from fuzzy functions are weighted according to the membership values and the output values are calculated as follows:

$$
\hat{y}_{k}=\frac{\sum_{i=1}^{c} \hat{y}_{i k} \mu_{i k}}{\sum_{i=1}^{c} \mu_{i k}}, i=1,2, \ldots, c, k=1,2, \ldots, n-p
$$

where, $\hat{y}_{i k}$ represents the predicted value obtained from $i^{t h}$ cluster for the observation $k$ and $\hat{y}_{k}$ is the forecasted value of the approach for the observation $k$. The flowchart of proposed method is given in Figure.1.

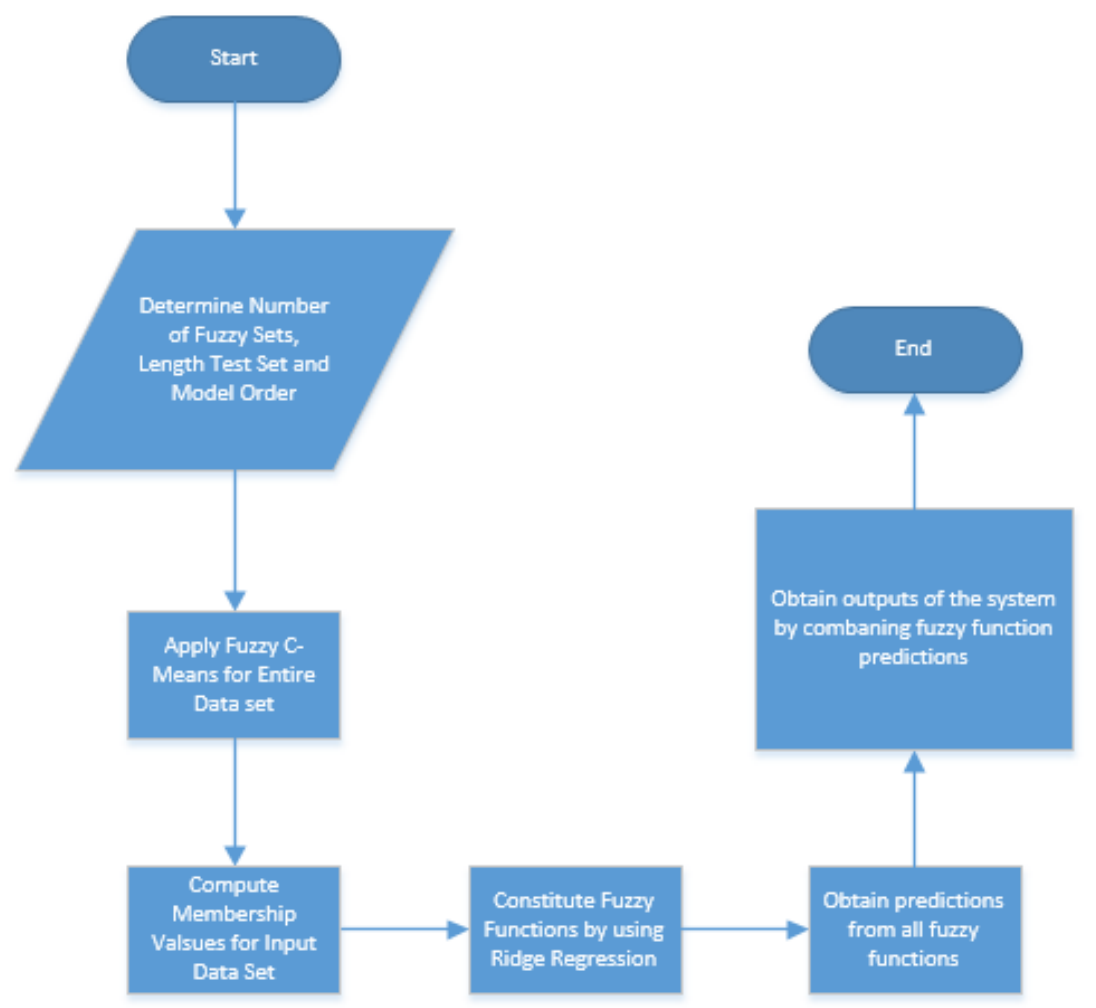

FIGURE 1. The flowchart of proposed method

\section{APPLICATIONS}

In this study, 12 time series data were analyzed for the evaluation of the forecasting performance of the proposed method. The first five time series data sets are Istanbul Stock Exchange Market (BIST100) data observed daily between years 2009 and 2013. The next five time series data set are Taiwan Stock Exchange Capitalization Weighted Stock Index (TAIEX) data observed daily between 2000 and 2004. The next data set is Australian Beer Consumption (AUST) data observed quarterly between years 1956 and 1994. Finally, the last data set is Turkey Electricity Consumption (TEC) data observed monthly between first month of 2002 and last month of 2013.

These time series and their features are presented in Table 1. The methods are compared in terms of Root Mean Square Error (RMSE) and Mean Absolute Percentage Error (MAPE) criteria. RMSE and MAPE are calculated using Equations (17) and (18):

$$
R M S E=\sqrt{\frac{1}{n} \sum_{k=1}^{n}\left(y_{k}-\widehat{y_{k}}\right)^{2}}
$$




$$
M A P E=\frac{1}{n} \sum_{k=1}^{n} \frac{\left|y_{k}-\hat{y}_{k}\right|}{y_{k}} * 100
$$

(18)

TABLE 1. The names and features of time series

\begin{tabular}{ccccccc}
\hline $\begin{array}{c}\text { Series } \\
\text { No }\end{array}$ & \multirow{2}{*}{ Series/Year } & \multicolumn{9}{c}{ The number of } & \multirow{2}{*}{-cut } & \multirow{2}{*}{ ntest } \\
\hline 1 & & Obs. & Lag & Clust & & \\
2 & BIST100/2009 & 103 & $1: 5$ & $3: 7$ & $0,0.1,0$. & 7,15 \\
3 & BIST100/2010 & 104 & $1: 5$ & $3: 7$ & $0,0.1,0$. & 7,15 \\
4 & BIST100/2011 & 106 & $1: 5$ & $3: 7$ & $0,0.1,0$. & 7,15 \\
5 & BIST100/2013 & 106 & $1: 5$ & $3: 7$ & $0,0.1,0$. & 7,15 \\
6 & TAIEX/2000 & 271 & $1: 5$ & $3: 7$ & $0,0.1,0$. & 7,15 \\
7 & TAIEX/2001 & 244 & $1: 5$ & $5: 15$ & $0,0.1,0$. & 47 \\
8 & TAIEX/2002 & 248 & $1: 5$ & $5: 15$ & $0,0.1,0$. & 43 \\
9 & TAIEX/2003 & 249 & $1: 5$ & $5: 15$ & $0,0.1,0$. & 43 \\
10 & TAIEX/2004 & 250 & $1: 5$ & $5: 15$ & $0,0.1,0$. & 43 \\
11 & AUST & 148 & 4,8 & $3: 10$ & $0,0.1,0$. & 16 \\
12 & TEC & 144 & $2: 16$ & $3: 10$ & $0,0.1,0$. & 12 \\
\hline
\end{tabular}

In the first case, the proposed method was implemented on BIST100 data sets. The results are compared with the results obtained by the following methods:

- ARIMA: Autoregressive Integrated Moving Average Model. The best model has been determined by Box-Jenkins Procedure.

- ES: Exponential Smoothing. Simple, Holt and Winters exponential smoothing methods were applied and the best model has been selected.

- MLP-ANN: Multi-Layer Artificial Neural Network. The number of hidden layer neurons varies between 1 and 5 and the best architecture was selected by trial\&error method. Levenberg Marquardt training algorithm was used as learning algorithm.

- SC: Song and Chissom time invariant fuzzy time series method (Song and Chissom, (1993b)). The number of fuzzy sets varies from 5 to 15 and the best number of fuzzy sets have been selected.

- FF: Fuzzy Function Approach (Turksen, (2008)). The model order and the number of fuzz sets varies from 1 to 5 and from 5 to 15 , respectively.

The best situations were determined for each series. Table 2 summarizes the results obtained from test set for Series 1-5.

TABLE 2. The obtained results for Series $1-5$ and when $n t e s t=7$ and 15

\begin{tabular}{ccccccccccccc}
\hline & \multicolumn{2}{c}{ ARIMA } & \multicolumn{2}{c}{ ES } & MLP-ANN & SC & & T1FF & T1FFRR \\
\hline Series/ & \multirow{2}{*}{ RMSE } & MAPE & RMSE & MAPE & RMSE & MAPE & RMSE & MAPE & RMSE & MAPE & RMSE & MAPE \\
Ntest & & & & & & & & & & & & \\
\hline Series 1/7 & 345 & 0.0087 & 345 & 0.0087 & 325 & 0.0083 & 140 & 0.0396 & 446 & 0.0101 & 319 & 0.0077 \\
Series & 540 & 0.0120 & 540 & 0.0120 & 525 & 0.0114 & 175 & 0.0438 & 534 & 0.0122 & 495 & 0.0112
\end{tabular}




\begin{tabular}{ccccccccccccc} 
Series 2/7 & 1221 & 0.0183 & 1208 & 0.0185 & 1077 & 0.0143 & 112 & 0.0182 & 1180 & 0.0179 & 1080 & 0.0155 \\
Series & 1612 & 0.0220 & 1612 & 0.0220 & 1603 & 0.0220 & 174 & 0.0265 & 1852 & 0.0264 & 1575 & 0.0213 \\
Series 3/7 & 1058 & 0.0144 & 1057 & 0.0144 & 920 & 0.0128 & 139 & 0.0200 & 1083 & 0.0153 & 915 & 0.0115 \\
Series & 1130 & 0.0150 & 1130 & 0.0150 & 1096 & 0.0146 & 136 & 0.0189 & 1146 & 0.0156 & 1028 & 0.0143 \\
Series 4/7 & 651 & 0.0084 & 651 & 0.0084 & 775 & 0.0111 & 129 & 0.0183 & 1034 & 0.0162 & 720 & 0.0106 \\
Series & 621 & 0.0088 & 621 & 0.0088 & 783 & 0.0117 & 104 & 0.0153 & 1038 & 0.0161 & 676 & 0.0100 \\
Series 5/7 & 1362 & 0.0116 & 1362 & 0.0116 & 1315 & 0.0109 & 145 & 0.0108 & 1512 & 0.0131 & 1251 & 0.0102 \\
Series & 1269 & 0.0109 & 1269 & 0.0109 & 1233 & 0.0107 & 193 & 0.0176 & 1279 & 0.0108 & 1237 & 0.0103 \\
\hline
\end{tabular}

The proposed method shows a $70 \%$ success for BIST 100 data sets and provides a noticeable improvement on the forecasting performance. Besides, although the proposed method has $30 \%$ failure, the multicollinearity problem has been eliminated. An example of multicollinearity problem in T1FF approach was given in Table 3 for Series 1 when ntest $=7$. It was shown that T1FF method has multicollinearity problem because some $\boldsymbol{V I F}$ values are greater than 10 . And also there is no multicollinearity problem when using T1FFRR method because all $\boldsymbol{V I F}$ values are less than 10 .

TABLE 3. The comparison of $\boldsymbol{V I F}$ values obtained from T1FF and T1FFRR methods for Series 1 when $n t e s t=7$.

\begin{tabular}{cc}
\hline \multicolumn{2}{c}{ VIF Values } \\
T1FF & T1FFRR \\
\hline 1963.121 & 2.225606 \\
1545.953 & 5.193898 \\
1368.769 & 5.433400 \\
1969.465 & 1.776532 \\
$4.01 \mathrm{E}-08$ & $3.85 \mathrm{E}-08$ \\
$6.97 \mathrm{E}-08$ & $6.73 \mathrm{E}-08$ \\
$6.81 \mathrm{E}-08$ & $6.68 \mathrm{E}-08$ \\
$6.74 \mathrm{E}-08$ & $6.41 \mathrm{E}-08$ \\
3.48E-08 & $3.47 \mathrm{E}-08$ \\
\hline
\end{tabular}

And also, the best situations for Series 1-5 for the best results were given in Table 4.

TABLE 4. The best situations for Series 1-5

\begin{tabular}{cccc}
\hline Series/Ntest & $m$ & cn & $\alpha$-cut \\
\hline Series $1 / 7$ & 4 & 5 & 0.1 \\
Series $1 / 15$ & 3 & 3 & 0.2 \\
Series $2 / 7$ & 4 & 3 & 0.1 \\
Series $2 / 15$ & 2 & 3 & 0.0 \\
Series 3/7 & 2 & 5 & 0.1 \\
Series 3/15 & 4 & 4 & 0.2 \\
Series 4/7 & 2 & 5 & 0.1 \\
Series 4/15 & 2 & 5 & 0.0 \\
Series 5/7 & 2 & 5 & 0.1 \\
Series 5/15 & 2 & 5 & 0.0 \\
\hline
\end{tabular}

In the second case, the proposed method was implemented on TAIEX data sets. Table 5 summarizes the results obtained from test set for Series 6-10. 
TABLE 5. All obtained results for TAIEX

\begin{tabular}{ccccccc}
\hline & \multicolumn{7}{c}{ Series } \\
\hline Methods & 6 & 7 & 8 & 9 & 10 & Mean \\
\hline Chen (1996) & 176.32 & 147.84 & 101.18 & 74.46 & 84.28 & 116.816 \\
Chen et al. (2010) & 129.42 & 113.33 & 66.82 & 53.51 & 60.48 & 84.712 \\
Chen and Chen & 123.62 & 115.33 & 71.01 & 58.06 & 57.73 & 85.15 \\
Chen et al. (2012) & 119.98 & 114.47 & 67.17 & 52.49 & 52.27 & 81.276 \\
T1FFRR & 119.73 & 113.17 & 62.55 & 48.73 & 51.66 & 79.168 \\
\hline
\end{tabular}

Analysis of Table 5 reveals that the proposed method exhibit more successful and superior forecasting performance compared to other methods in terms of RMSE performance criteria. And also, the best situations for Series 6-10 for the best results are given in Table 6.

TABLE 6. The best situations for Series 6-10

\begin{tabular}{cccc}
\hline Series & $m$ & cn & $\alpha$-cut \\
\hline Series 6 & 5 & 9 & 0.1 \\
Series 7 & 2 & 9 & 0.2 \\
Series 8 & 4 & 13 & 0.1 \\
Series 9 & 2 & 5 & 0.2 \\
Series 10 & 4 & 11 & 0.0 \\
\hline
\end{tabular}

In the next case, the proposed method was implemented on AUST data set (Series11). Table 7 summarizes the results obtained for the test set for Series11. Series 11 was forecasted by Seasonal Autoregressive Integrated Moving Average (SARIMA), Winters' multiplicative exponential smoothing method (WMES), linear and nonlinear artificial neural network model (L\&NL-ANN) proposed by Yolcu et al. (2013), multiplicative neuron model based fuzzy time series method (MNM-FTS) proposed by Aladag (2013) and T1FF method.

TABLE 7. All obtained results for Series 11

\begin{tabular}{ccccccc}
\hline Test Data & SARIMA & WMES & $\begin{array}{c}\text { Yolcu et al. } \\
\mathbf{( 2 0 1 3 )}\end{array}$ & $\begin{array}{c}\text { Aladag } \\
\mathbf{( 2 0 1 3 )}\end{array}$ & T1FF & T1FFRR \\
\hline 430.50 & 452.72 & 453.91 & 449.92 & 437.50 & 446.20 & 446.64 \\
600.00 & 578.29 & 575.22 & 574.28 & 537.50 & 580.12 & 580.95 \\
464.50 & 487.70 & 502.32 & 481.47 & 437.50 & 483.04 & 481.19 \\
423.60 & 446.28 & 444.73 & 442.79 & 437.50 & 442.97 & 442.76 \\
437.00 & 456.77 & 459.66 & 445.12 & 437.50 & 444.74 & 445.13 \\
574.00 & 583.51 & 582.48 & 571.97 & 537.50 & 579.90 & 579.87 \\
443.00 & 492.13 & 508.64 & 472.76 & 487.50 & 468.01 & 465.80 \\
410.00 & 450.36 & 450.31 & 416.36 & 437.50 & 418.98 & 418.72 \\
420.00 & 461.01 & 465.40 & 428.63 & 437.50 & 431.60 & 431.85 \\
532.00 & 588.96 & 589.74 & 559.89 & 562.50 & 559.41 & 559.10 \\
432.00 & 496.77 & 514.96 & 445.75 & 462.50 & 444.08 & 442.34
\end{tabular}




\begin{tabular}{ccccccc}
420.00 & 454.64 & 455.89 & 390.25 & 412.50 & 394.99 & 394.65 \\
411.00 & 465.46 & 471.15 & 412.38 & 437.50 & 409.72 & 410.03 \\
512.00 & 594.71 & 597.00 & 533.19 & 537.50 & 525.60 & 525.92 \\
449.00 & 501.67 & 521.28 & 442.13 & 437.50 & 438.91 & 436.09 \\
382.00 & 459.17 & 461.46 & 405.08 & 412.50 & 409.07 & 408.81 \\
\hline RMSE & 47.0367 & 53.3295 & 18.7888 & 29.1381 & 17.3926 & 17.0845 \\
MAPE & 0.0949 & 0.1072 & 0.0357 & 0.0532 & 0.0345 & 0.0340 \\
\hline
\end{tabular}

Analysis of Table 7 reveals that the proposed method exhibit more successful and superior forecasting performance compared to other methods in terms of MAPE and RMSE performance measures. We conclude that the best result is obtained in the case where " $m=8, c n=5$, and $\alpha-$ $c u t=0 "$. In Table 7, the results of methods compared with the proposed method are taken from [20].

The graph of the real observations and the forecasts obtained from proposed method for the test set was presented in Figure. 2. According to this graph, it is clearly seen that the forecasts obtained from proposed method are very accurate.

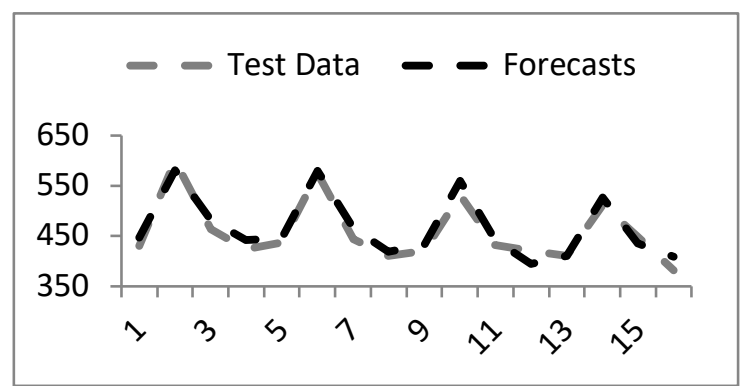

FIGURE 2. The graph of the real observations and the forecasts obtained from proposed method for Series 11

Finally, the proposed method was implemented to TEC data set (Series 12). The data set was forecasted by using SARIMA, MLP-ANN, Multiplicative Neuron Model Artificial Neural Network (MNM-ANN), L\&NL-ANN, and T1FF approach.

Table 8 summarizes the results obtained from test set for Series 12. Table 8 reveals that the proposed method exhibit more successful and superior forecasting performance compared to other methods in terms of MAPE and RMSE performance measures. We conclude that the best result is obtained in the case where " $m=16, c n=4$, and $\alpha-c u t=0.1 "$.

TABLE 8. All obtained results for Series 12

\begin{tabular}{ccccccc}
\hline Test Data & MLP-ANN & $\begin{array}{c}\text { SARIMA } \\
(\mathbf{0 , 1 , 1 ) ( 0 , 1 , 1 ) 1 2}\end{array}$ & MNM-ANN & L\&NL-ANN & T1FF & $\begin{array}{c}\text { Proposed } \\
\text { Method }\end{array}$ \\
\hline 21275408487 & 21504260392 & 21690314907 & 21929976335 & 22005935580 & 21666285710 & 21675649037 \\
18841712637 & 21407932217 & 19879748318 & 19674336447 & 21273737442 & 20685602131 & 20715993541 \\
20463933683 & 19934741061 & 20679491118 & 20759353666 & 20386359964 & 19996267563 & 20048429544 \\
19139248871 & 17339157313 & 18616749529 & 18177421217 & 19068902087 & 17987700997 & 18030742336 \\
19511728912 & 19132107582 & 18166751109 & 19502618441 & 19337714416 & 19394021620 & 19427466506 \\
20132602347 & 20521392811 & 19476718063 & 19961628854 & 20493224715 & 20584842565 & 20555636973 \\
22648523194 & 21559624073 & 22996373189 & 21466603203 & 22493619106 & 22702026988 & 22707403125
\end{tabular}




\begin{tabular}{ccccccc}
21698207982 & 21608712088 & 22602364226 & 20970484983 & 22642925619 & 21714016063 & 21762357930 \\
20358717408 & 20450417618 & 19372692274 & 20819409088 & 20387962879 & 19965826638 & 20025776421 \\
18964661109 & 19608790983 & 19145368564 & 18842139533 & 19384311943 & 18624614607 & 18648025760 \\
20061232838 & 20798229864 & 18281990717 & 20341486661 & 20668793095 & 20140094911 & 20160893745 \\
22405662577 & 21396071971 & 21319220705 & 20523920152 & 22329629382 & 22138069735 & 22109509894 \\
\hline RMSE & 1065870606 & 917321409.3 & 813259007.7 & 820978567.5 & 687192130.1 & 681722004.5 \\
MAPE & 0.039841988 & 0.038842 & 0.030114538 & 0.025444349 & 0.023662007 & 0.023219348 \\
\hline
\end{tabular}

And also, the graph of the real observations and the forecasts obtained from proposed method for the test set was presented in Figure. 3. According to this graph, it is clearly seen that the forecasts obtained from proposed method are very accurate.

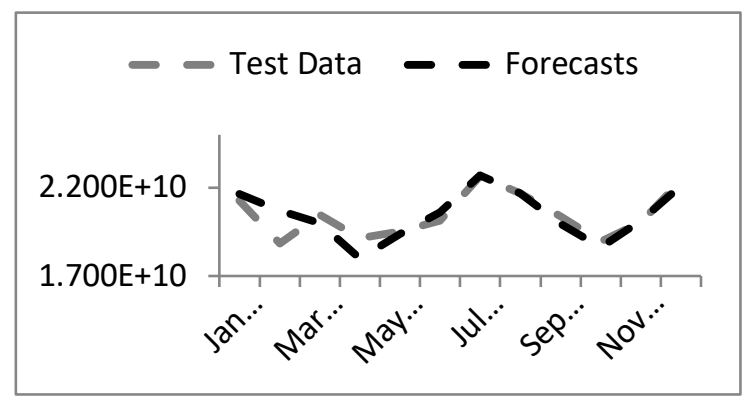

FIGURE 3. The graph of the real observations and the forecasts obtained from proposed method for Series 12 .

\section{CONCLUSION AND DISCUSSIONS}

Fuzzy function approach is a kind of fuzzy inference system that can produce successful results for the analyzing of forecasting problems. In fuzzy function approach, a fuzzy function corresponding to each fuzzy set is generated by using multiple regression analysis. A high correlation can be observed between the non-linear aspect of membership functions and the multiple linear regression method used to define fuzzy functions which have multicollinearity problem.

In order to overcome this problem, a new fuzzy function approach using ridge regression instead of multiple linear regression method in Type 1 fuzzy function approach is proposed. The multicollinearity problem was eliminated by using our new proposed method. In order to show the superior forecasting performance of the proposed method, we applied and tested it on several real-world data sets.

In future studies, the new proposed approach can be applied to Type 2 fuzzy function approach and also different regression techniques can be used for Type 1 fuzzy function.

\section{REFERENCES}

ALADAG, C. H., TURKSEN, I. B., DALAR, A. Z., EGRIOGLU, E., and YOLCU, U. 2014. Application of Type-1 fuzzy functions approach for time series forecasting. Turkish Journal of Fuzzy Systems, 5(1): 1-9.

ALADAG, C.H. 2013. Using multiplicative neuron model to establish fuzzy logic relationships. Expert Systems with Applications, 40 (3): 850 - 853.

BEYHAN, S., and ALCI, M. 2010. Fuzzy functions based ARX model and new fuzzy basis function 
models for nonlinear system identification. Applied Soft Computing, 10: 439-444.

BEZDEK, J.C. (1981) Pattern recognition with fuzzy objective function algorithms. Plenum Press, NewYork, USA.

CELIKYILMAZ, A. and TURKSEN, I. B. 2008b. Uncertainty modeling of improved fuzzy functions with evolutionary systems. IEEE Trans. On system, Man and Cybernetics, 38(4): 1098-1110.

CELIKYILMAZ, A. and TURKSEN, I. B. Modeling uncertainty with Fuzzy Logic, Studies in Fuzziness and Soft Computing Springer. 2009.

CELIKYILMAZ, A., and TURKSEN, I. B. 2008a. Enhanced fuzzy system models with improved fuzzy clustering algorithm. IEEE Trans. Fuzzy Syst., 16(3): 779-794.

CHEN, S. M., 1996. Forecasting enrollments based on fuzzy time-series. Fuzzy Sets and Systems, 81: 311-319.

CHEN, S. M., and CHANG, Y.C. 2010. Multi-variable fuzzy forecasting based on fuzzy clustering and fuzzy rule interpolation techniques. Inf. Sci., 180(24): 4772-4783.

CHEN, S. M., and CHEN, C. D. 2011. TAIEX forecasting based on fuzzy time series and fuzzy variation groups. IEEE Trans. Fuzzy Syst., 19(1): 1-12.

CHEN, S. M., CHU, H. P., and SHEU, T. W. 2012. TAIEX forecasting using fuzzy time series and automatically generated weights of multiple factors, IEEE Transactions On Systems, Man, and Cybernetics—Part A: Systems and Humans, 42(6): 1485-1495.

HOERL A. E., KENNARD, R. W. and BALDWIN, K. F. 1975. Ridge regression: some simulations. Communications in Statistics, 4: 105-123.

HOERL, A. E., and KENNARD, R. W. 1970a. Ridge regression: biased estimation for nonorthogonal problems. Technometrics, 12: 55-67.

JANG, J. S. R. 1993. ANFIS: Adaptive network based fuzzy inference system. IEEE Trans. On system, Man and Cybernetics, 23(3): 665-685.

SONG, Q. and CHISSOM, B. S. 1993b. Forecasting enrollments with fuzzy time series - Part I. Fuzzy Sets and Systems, 54: 1-10.

TAKAGI, T., and SUGENO, M. 1985. Fuzzy identification of systems and its applications to modeling and control. Man and Cybernetics, 15:116-132.

TURKSEN, I. B. 2009. Fuzzy system models. Encyclopedia Complex. Syst. Sci. 4080-4094.

TURKSEN, I.B. 2008. Fuzzy function with LSE. Applied Soft Computing, 8: 1178-1188.

YOLCU, U., ALADAG, C. H., and EGRIOGLU, E. 2013. A new linear \& nonlinear artificial neural network model for time series forecasting. Decision Support System Journals, 54: 1340-1347.

ZARANDI, M. H. F., ZARINBAL, M. GHANBARI, N. and TURKSEN, I. B. 2013. A new fuzzy functions model tuned by hybridizing imperialist competitive algorithm and simulated annealing, Application: Stock price prediction. Information Sciences, 222 (10): 213-228. 